\title{
El proceso docente de adquisición de habilidades clínicas en la Universidad de Córdoba: una autoevaluación de los alumnos
}

\author{
J ulián Torre-Cisneros, Angela Camacho, J osé López-Miranda, Pedro Sánchez-Guijo, \\ Francisco Pérez J iménez.
}

Facultad de Medicina. Universidad de Córdoba. Córdoba

Introducción. Durante los estudios de pregrado se realiza un proceso continuo de adquisición del hábito de la competencia profesional como médico general. Para que esta formación fuese eficaz seria deseable establecer las habilidades clínicas exigibles y el nivel de competencia requerido en un postgraduado. Estos objetivos deben ser tenidos en cuenta para planificar los métodos docentes y de evaluación de competencias. Material y métodos. Como parte del proceso de definición de las habilidades clínicas que deberán ser incluidas en los objetivos docentes de nuestra Facultad, hemos realizado una autoevalución de los alumnos que finalizan el pregrado referida a la anamnesis, exploración física y procedimientos clínicos básicos. Resultados. Los resultados indican que, a juicio de los estudiantes, las habilidades clínicas consideradas han sido adquiridas por un insuficiente numero de alumnos. A título de ejemplo, el $17 \%$ de los alumnos no se encuentra capacitado para realizar una anamnesis centrada en el paciente.

Conclusiones. La subjetividad del método obliga a completar los resultados con los obtenidos en una evaluación objetiva de habilidades. Sin embargo permiten intuir que es preciso modificar el método docente de la enseñanza práctica y la evaluación de competencias clínicas en nuestra Facultad.

Palabras clave: competencia profesional, objetivos, habilidades, evaluación.

Correspondencia:

Prof. Francisco Pérez J iménez.

Facultad de Medicina.

Avda. Menéndez Pidal sn. 14004-Córdoba.

E-mail: fperezjimenez@uco.es
The acquisition of clinical skills at the University of Cordoba: a student self-assessment.

Introduction: During undergraduate study, ongoing training is needed to acquire professional competence as a general practitioner. For this training to be effective, it is necessary to establish the clinical skills and professional competence required for postgraduate students. These objectives must be taken into consideration when defining teaching methods and assessing competence.

Material and methods. With the aim of identifying which clinical skills should be included in the educational goals of our Faculty, graduating students were asked to assess their competence in conducting anamneses, physical examinations and basic clinical skills and procedures.

Results. Our findings suggest that too few students believe they have acquired the above skills. Seventeen percent of the students do not consider themselves to be qualified to conduct patient anamnesis. Conclusions. Due to the subjectivity of the method, the results must be completed through an objective assessment of the skills acquired by our students.

However, our study also suggests that it is necessary to change the practical teaching methods currently in use and the manner in which clinical competence is assessed in our Faculty.

Key words: professional competence, objectives, skills, assessment. 


\section{INTRODUCCION}

El aprendizaje debe concebirse como "un proceso continuo de adquisición del hábito de la competen cia profesi onal", en este caso de la competencia como médico general ${ }^{1-4}$. Este proceso comienza en el primer curso de medicina y continua durante toda la vida profesional por medio de la formación continuada. La "continuidad" del proceso docente se basa en la adquisición de diferentes "niveles de compe tencia" que exigen la definición de objetivos determinados, el diseño del método docente apropiado a los objetivos propuestos y una evaluación dirigida a todas las áreas de competencia induidas en los objetivos de cada nivel. El conjunto de esta actividad es lo que denominamos "docencia".

La "competencia profesional" como médico consiste en el uso habitual y juicioso de conocimientos, habilidades (entre las que destaca las habilidades clínicas) y actitudes, que es preciso desarrollar en la práctica clínica diaria en beneficio del paciente y de la comunidad a la que se sirvet. Entre los objetivos docentes de la licenciatura de medicina deberían establecerse las habilidades dínicas exigibles y el nivel de competencia requerido en un postgradua$\mathrm{do}^{5-12}$. La Facultad de Medicina de la U niversidad de Córdoba ha comenzado un proceso de definición de objetivos institucionales y departamentales que dirijan su actividad docente (método docente y evaluación) en los próximos años. Con el convencimiento de que la evaluación es el "motor del aprendizaje" estos objetivos deberán ser tenidos en cuenta para planificar los métodos de evaluación ${ }^{10-12}$. Como parte de este proceso hemos realizado la autoevalución contenida en este trabajo. Con ella pretendemos tener una visión de lo que el alumno percibe que ha aprendido en el periodo de licenciatura. Una vez completada esta autoevaluación con la opinión experta del profesorado tendremos una base sólida para modificar el método docente empleado para conseguir los objetivos propuestos.

\section{MATERIAL Y METODOS}

Definición de las habilidades clínicas y procedi mientos incluidos en la autoevaluación. Las habilidades clínicas y procedimientos induidos en la encuesta se basó en la utilizada en trabajos previamente publicados en nuestro país ${ }^{13}$, con modificaciones para adaptarlos a las directrices utilizadas en el proceso de definición de competencias de la Facultad de Medicina de la U niversidad de Córdoba

TABLA 1: Habilidades clínicas consideradas en la autoevaluación.

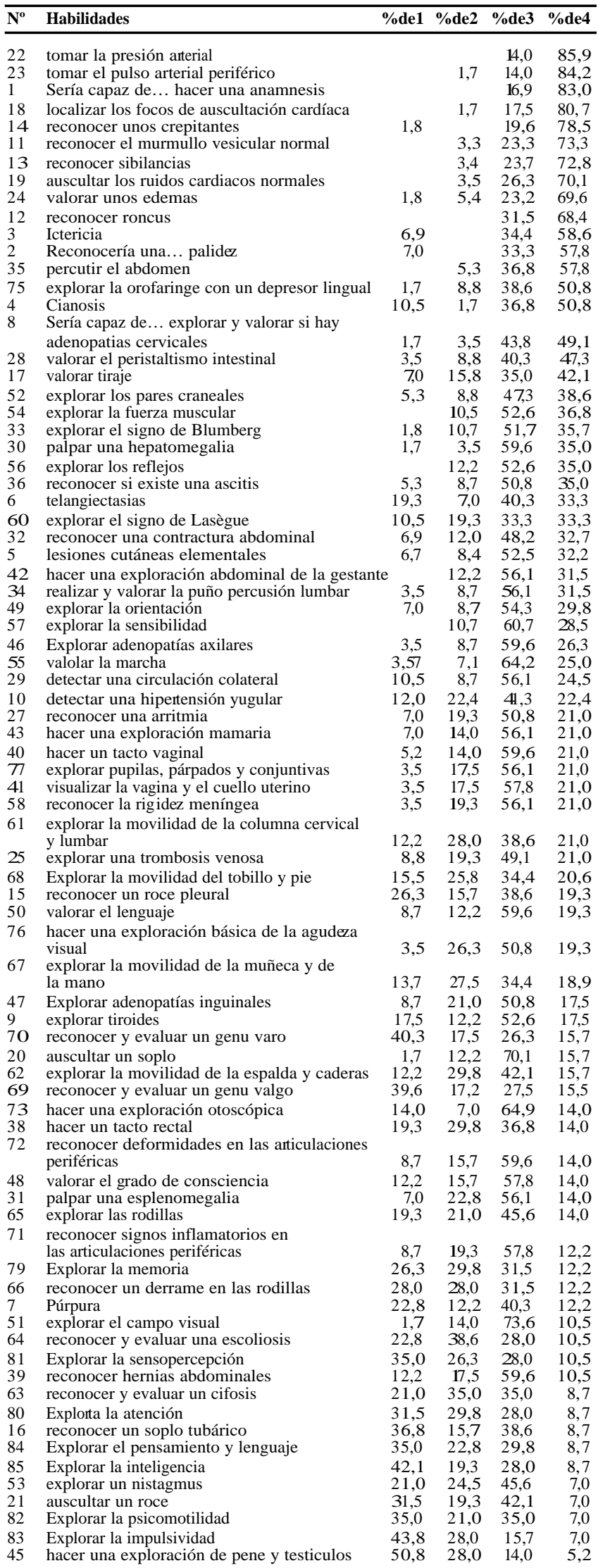




\begin{tabular}{llllll}
88 & Explorar el comportamiento & 36,8 & 26,3 & 24,5 & 5,2 \\
78 & Explorar el fondo de ojo & 10,5 & 10,5 & 73,6 & 5,2 \\
87 & Explorar la personalidad & 43,8 & 22,8 & 21,0 & 5,2 \\
86 & Explorar la afectividad & 41,3 & 24,1 & 24,1 & 5,1 \\
37 & reconocer si existe un globo vesical & 31,5 & 21,0 & 40,3 & 3,5 \\
59 & explorar un enfermo en coma & 45,6 & 21,0 & 26,3 & 3,5 \\
26 & explorar una oclusión arterial & 36,8 & 26,3 & 31,5 & 1,7 \\
74 & hacer una exploración acumétrica & 29,8 & 36,8 & 24,5 & 1,7 \\
44 & hacer una exploración prostática & 52,6 & 26,3 & 19,3 & \\
\hline
\end{tabular}

NOTA. 1: sé lo que es y me lo han explicado. 2: lo he visto hacer. 3: lo he practicado. 4: sería capaz de realizarlas siempre y en cualquier circunstancia.

TABLA 2: Procedimientos clínicos considerados en la autoevaluación.

\begin{tabular}{|c|c|c|c|c|c|}
\hline & Procedimientos & \multicolumn{4}{|c|}{ \%de1 \%de2 \%de3 \%de4 } \\
\hline 89 & Sería capaz de... tomar la presión arterial & 3,5 & 10,5 & 85,9 & \\
\hline 90 & tomar el pulso arterial & & 1,7 & 17,5 & 80,7 \\
\hline 137 & Usar el correo electrónico & 3,5 & 10,5 & 22,8 & 61,4 \\
\hline 107 & interpretar los resultados de los exámenes & & & & \\
\hline & analíticos básicos de sangre y orina & 1,7 & 3,5 & 43,8 & 50,8 \\
\hline 108 & interpretar la radiología simple de tórax & 1,7 & 1,7 & 52,6 & 43,8 \\
\hline 142 & Hacer un parte de lesiones al juzgado & 10,5 & 21,0 & 21,0 & 43,8 \\
\hline 109 & interpretar la radiología simple de abdomen & & 3,5 & 56,1 & 40,3 \\
\hline 112 & aplicar medidas de asepsia en heridas simples & 3,5 & 14,0 & 42,1 & 40,3 \\
\hline 110 & interpretar una fractura de hueso largo & 1,7 & 10,5 & 50,8 & 36,8 \\
\hline 106 & efectuar una tira básica de orina & 7,0 & 28,0 & 29,8 & 33,3 \\
\hline 91 & interpretar un electrocardiograma & 3,5 & 1,7 & 61,4 & 31,5 \\
\hline 105 & determinar glucemias capilares & 19,3 & 24,5 & 24,5 & 28,0 \\
\hline 134 & hacer búsquedas en Medline (PubMed) & 8,7 & 8,7 & 56,1 & 24,5 \\
\hline 95 & realizar una otoscopia & 8,7 & 14,0 & 54,3 & 22,8 \\
\hline 138 & Informar al paciente de de su curso clínico & 5,2 & 28,0 & 40,3 & 22,8 \\
\hline 114 & realizar curas cutáneas & 5,2 & 31,5 & 40,3 & 22,8 \\
\hline 139 & Informar a la familia del curso clínico del paciente & te 1,7 & 31,5 & 40,3 & 21,0 \\
\hline 141 & Solicitar un consentimiento informado & 3,4 & 43,1 & 31,0 & 18,9 \\
\hline 111 & interpretar una fractura vertebral & 7,5 & 18,8 & 54,7 & 18,8 \\
\hline 133 & hacer búsquedas en bases bibliográficas & & & & \\
\hline & basadas e & 20,0 & 10,9 & 47,2 & 18,1 \\
\hline 113 & colocar suturas en heridas simples & 12,2 & 24,5 & 43,8 & 17,5 \\
\hline 130 & Administrar inyectables subcutáneo & 17,5 & 38,6 & 26,3 & 14,0 \\
\hline 140 & $\begin{array}{l}\text { Informar a la familia de una mala noticia } \\
\text { (muerte cáncer) }\end{array}$ & & & & \\
\hline 119 & hacer el primer tratamiento de una hemorragia & & נינה & 1,0 & $2, r$ \\
\hline & externa & 31,5 & 38,6 & 15,7 & 12,2 \\
\hline 129 & istrar inyectables intramusculares & 15,7 & 42,1 & 26,3 & 12,2 \\
\hline 128 & Administrar insulina & 24,5 & 40,3 & 19,3 & 12,2 \\
\hline 94 & Administrar oxigenoterapia & 19,3 & 45,6 & 24,5 & 10,5 \\
\hline 115 & realizar la RCP básica & 43,8 & 28,0 & 17,5 & 10,5 \\
\hline 136 & Realizar un informe clínico & 8,7 & 36,8 & 38,6 & 8,7 \\
\hline 135 & Presentar un caso clínico & 3,5 & 36,8 & 43,8 & 8,7 \\
\hline 117 & mantener una ventilación correcta & & & & \\
\hline 97 & $\begin{array}{l}\text { de la vía aérea } \\
\text { realizar un taponamiento anterior }\end{array}$ & 42,1 & 36,8 & 14,0 & 7,0 \\
\hline & de una epistaxis & 45,6 & 24,5 & 19,3 & 7,0 \\
\hline 118 & & 38,6 & 40,3 & 15,7 & 5,2 \\
\hline 104 & realizar una $\mathrm{p}$ & 31,5 & 38,6 & 21,0 & 5,2 \\
\hline 132 & Administrar i & 25,0 & 37,5 & 30,3 & 3,5 \\
\hline 131 & Administrar inyectables intravenosos & 17,5 & 56,1 & 19,3 & 3,5 \\
\hline 127 & colocar sondas vesicales & 29,8 & 45,6 & 15,7 & 3,5 \\
\hline 124 & colocar un collarín cervical & 35,0 & 40,3 & 14,0 & 3,5 \\
\hline 103 & $\begin{array}{l}\text { extraer cuerpos extraños superficiales } \\
\text { del globo ocular }\end{array}$ & 280 & & & 35 \\
\hline 123 & realizar una inmovilización elástica & 20,0 & & נינד & 3,5 \\
\hline & del tobillo y pie & 40,3 & 33,3 & 17,5 & 3,5 \\
\hline 93 & utilizar inhaladores y aerosoles & 10,5 & 49,1 & 36,8 & 3,5 \\
\hline 126 & colocar sondas nasogástricas & 33,3 & 45,6 & 14,0 & 3,5 \\
\hline 96 & hacer una laringosocopia indirecta sobre & & & & \\
\hline & & 53,5 & 19,6 & 14,2 & 1,7 \\
\hline 116 & realizar la $\mathrm{RCP}$ a & 55,3 & 26,7 & 16,0 & 1,7 \\
\hline 102 & medir la tensión & 17,8 & 41,0 & 35,7 & 1,7 \\
\hline 99 & utilizar un espejo frontal simple & 42,1 & 19,3 & 33,3 & 1,7 \\
\hline 125 & colocar vías intravenosas & 28,0 & 47,3 & 19,3 & 1,7 \\
\hline 122 & na inmovilización elástica de la $n$ & & & & \\
\hline & y la $n$ & 42,1 & 35,0 & 17,5 & 1,7 \\
\hline 92 & realizar un peak-flow & 43,8 & 15,7 & 19,3 & 1,7 \\
\hline 121 & realizar una inmovilización provisional con yeso & & & & \\
\hline & del miembro inferior & 45,6 & 35,0 & 14,0 & 1,7 \\
\hline 120 & $\begin{array}{l}\text { realizar una inmovilización provisional } \\
\text { con yeso del miembro superior }\end{array}$ & 5.6 & ד, & & \\
\hline 98 & realizar un neumotaponamiento de & & & & \\
\hline & & 71,9 & 14,0 & 8,7 & \\
\hline 100 & $\begin{array}{l}\text { extraer cuerpos extraños de la garganta, } \\
\text { fosas nasales y los oidos }\end{array}$ & 59,6 & 26,3 & 10,5 & \\
\hline 101 & extraer cuerpos extraños de la laringe & 66,6 & 22,8 & 5,2 & \\
\hline
\end{tabular}

NO TA. 1: sé lo que es y me lo han explicado. 2: lo he visto hacer. 3:

lo he practicado. 4: sería capaz de realizarlas siempre y en cualquier circunstancia.
(Tabla 1 y 2). Este proceso, impulsado desde el Decanato de la Facultad esta basado en las competencias definidas por el Accreditatión Council for Graduate Medical Education (USA), la reforma 2001 del Core Clerkship in Internal Medicine (SGIM/CDIM Project) promovido por la Society of General Internal Medicine (USA) y los Clerkship Directors in Internal Medicine de USA, por The Scottish Deans' Medical Curriculum Group, por el American Board of Internal Medicine (2001), y por el Blueprint 1994: training of doctors in the Netherlands ${ }^{1419}$.

Valoración de las encuestas. Las encuestas pre viamente definidas se repartieron a todos los alumnos del último curso de la Licenciatura evaluados en la asignatura de Patología Médica III en la convocatoria ordinaria de J unio de 2002 (90 alumnos). La participación fue voluntaria y no se preguntaron los motivos para no realizar la encuesta. En cada una de las habilidades o procedimientos se propusieron las siguientes respuestas: 1 . sé lo que es y me lo han explicado; 2. Io he visto hacer; 3. Io he practicado; 4. sería capaz de realizarlas siempre y en cualquier circunstancia.

Contexto en el que se ha realizado la encuesta. EI Plan de Estudios de Medicina de la UCO es un plan por asignaturas no integradas aprobado (B.O.E. de 3 de Febrero de 1996). Se configura con 508 créditos, estructurados en dos ciclos: un primer ciclo de 3 años con 250 créditos y un segundo cido de otros tres años con 258 créditos. Tiene gran peso las materias troncales de obligatoria indusión en todos los planes de estudio de la Licenciatura de Medicina (408 créditos, 205 créditos en el primer ciclo y 203 créditos en el segundo cido). De todos los créditos de materias troncales del segundo ciclo, 124 créditos son de prácticas dínicas. Por el contrario no se han establecido materias obligatorias propias de la Universidad de Córdoba. Contempla 49 créditos de materias optativas libremente establecidas por la Universidad de Córdoba para que el alumno escoja entre ellas ( 21 créditos en el primer cido y 28 créditos en el segundo ciclo). Su implantación comenzó en el curso académico 1996-1997, entrando su implantación en el curso académico 2001-2002 en el curso 6ㅇ (Cido 2ㅇ).

Registro y análisis de las encuestas. El registro de las encuestas se realizó con la ayuda de la aplicación Access 2000 de Microsoft. La ordenación, recuento y estimación porcentual de cada uno de las cuestiones se realizaron con la ayuda de las utilidades de dicha aplicación y de la hoja de cál culo Excel 


\begin{tabular}{|c|c|c|c|c|}
\hline CATEGORÍA & $\%$ de 1 & $\%$ de 2 & $\%$ de 3 & $\%$ de 4 \\
\hline CARDIOV ASCUL AR & 3,5 & 2,3 & 29,8 & 66,0 \\
\hline R ADIOLOGIA & 3,6 & 8,6 & 53,5 & 34,9 \\
\hline LABORATORIO & 14,9 & 23,6 & 29,8 & 29,3 \\
\hline COM UNICACIÓN & 5,9 & 32,3 & 32,4 & 24,7 \\
\hline AUTOAPREN DIZAJE & 14,3 & 9,8 & 51,7 & 21,3 \\
\hline CIRUGIA GENERAL & 29,0 & 30,1 & 25,6 & 14,7 \\
\hline ORL & 49,7 & 20,1 & 20,8 & 8,3 \\
\hline TECNICAS MANUALES & 23,9 & 44,1 & 21,3 & 6,8 \\
\hline RESPI RATORIO & 24,5 & 36,8 & 26,9 & 5,2 \\
\hline OFTALMOLOGIA & 22,9 & 36,3 & 33,6 & 2,6 \\
\hline TRAU MATOLOGIA & 41,7 & 35,0 & 16,1 & 2,4 \\
\hline
\end{tabular}

NOTA 1: sé lo que es y me lo han explicado. 2: lo he visto hacer. 3: lo he practicado. 4: sería capaz de realizarlas siempre y en cualquier circunstancia.

2000. Asimismo, para el análisis estadístico y el contraste de proporciones se utilizó la utilidad Medcalc (versión 6.16, demo: http://www.medcalc.be).

\section{RESULTADOS}

Participaron vduntariamente en la encuesta 59 al umnos de los que 22 er an varones. Las edades de los encuestados osailaban entre 23 y 28 años. La distribución de datos (porcentajes de respuesta) no se ajustó, como era de esperar, a la normalidad (Kol mogorovSmirnov $\varangle 0,001 ; x^{2} \varangle 0,0001$ ), ni por separado (habilidades y procedimientos) ni conjuntamente, lo que no permiteel uso dela estadística paramétrica. Nohubo difer encias estadísticamente significativas entre $\mathrm{d}$ grado de respuesta a unas y a otras preguntas, ni por separado ni juntas (Kruskal-Wallis: estadístico $\mathrm{H}=$ 136,8; $p=0,4839$ ). Por tanto, los resultados de esta encuesta tienen un val or descriptivo.

EI número de alumnos que respondieron " 4 " a los distintos ítems, en porcentaje del total de encuestas, y por orden decreciente, se especifican en la Tabla 1 y en la Tabla 2.

La Tabla 3 y la Tabla 4 recogen los porcentajes medios de respuestas por grupos de habilidades (sistema) o procedimientos (categoría).

EI $83 \%$ de los alumnos admiten que serían capaces de realizar una anamnesis completa y correcta. Los grupos de habilidades (Tabla 3) de exploración mejor adquiridas según las encuestas fueron las referidas a respiratorio, cardiovascular y cutáneo. Las peores fueron las correspondientes a psiquiatría (8\%). Pese a lo anterior, la habilidad aislada que los alumnos parecen haber aprendido peor es la realización de una exploración prostática (Tabla 1), seguida de la realización de acumetrías o la exploración de una odusión arterial.
Por grupos de procedimientos (Tabla 4), aquellos con un porcentaje más alto de respuesta fueron los de cardiovascular (66\%), estando todos los demás (radiología, comunicación, laboratorio...) muy por debajo (menos del 35\%). Los peor adquiridos fueron los grupos de traumatología, oftalmología, respiratorio y técnicas manuales (todos ellos por debajo del 8\%). Sin embargo, si observamos el porcentaje de los ítems independientes (no por grupos), los procedi mientos (Tabla 2) peor adquiridos son el neumotaponamiento posterior y la extracción de cuerpos extraños de localización ORL, a los que no ha respondido ningún estudiante la opción 4. Pero, además, hay otros procedimientos a los que responden 4 solo 1 o 2 alumnos, como por ejemplo, alguno correspondiente a traumatología (colocar yesos o inmovilizaciones elásticas), ORL (uso del espejo frontal) u oftalmología (toma de tensión ocular), técnicas manuales como la realización de una RCP avanzada, realizar laringoscopia o colocar sondas nasogástricas, etc. Casi un $20 \%$ de los alumnos encuestados no responde nada al ítem no 92 ("realizar un peak-flow"). Por el contrario, los mejor adquiridos fueron la toma de la tensión arterial y el pulso arterial periférico y el uso del correo electrónico.

De forma global, y en valores absolutos, la tasa de respuestas " 4 " de las habilidades (27\%) es superior a la de procedimientos (17\%), aunque la diferencia no es estadísticamente significativa $\left(x^{2}=1,322\right.$; grados de libertad $=1 ; p=0,2502$ ).

\section{DISCUSIÓN}

Los resultados obtenidos reflejan sólo lo que el alumno "piensa que sabe hacer" pero no lo que el alumno "sabe cómo hacer" o realmente "hace". Sin

\begin{tabular}{|c|c|c|c|c|}
\hline SISTEMAS & $\%$ de 1 & $\%$ de 2 & $\%$ de 3 & $\%$ de 4 \\
\hline ANAM NESIS & & & 16,9 & 83,0 \\
\hline RESPI RATORIO & 17,9 & 10,8 & 30,0 & 51,9 \\
\hline CARDIOVASCUL AR & 14,6 & 12,1 & 33,9 & 45,7 \\
\hline CUTANEO & 12,2 & 7,3 & 39,6 & 40,8 \\
\hline CABEZA Y CUELLO & 10,4 & 12,7 & 45,9 & 29,6 \\
\hline DIGES TIVO & 9,4 & 13,1 & 49,4 & 28,5 \\
\hline NERVIOSO & 12,0 & 13,7 & 54,2 & 22,4 \\
\hline ORL & 15,2 & 17,5 & 42,6 & 22,2 \\
\hline ADENOPATIAS & 6,1 & 14,9 & 55,2 & 21,9 \\
\hline GEN ITAL & 23,8 & 18,7 & 43,8 & 20,0 \\
\hline LOCOMOIOR & 19,4 & 24,8 & 38,0 & 16,3 \\
\hline OFTALMOLOGIA & 5,8 & 18,1 & 60,2 & 15,2 \\
\hline PSIQU IATRIA & 37,1 & 25,0 & 26,6 & 7,8 \\
\hline
\end{tabular}


embargo esta información es una valiosa herramienta para la personalización de objetivos basados en técnicas de "empowerment" ${ }^{20}$.

EI proceso de definición de competencias profesionales que se desarr olla en nuestra Facultad afecta a conocimi entos, habili dades y actitudes. Induye el desarrollo de una lista de habilidades y procedimientos dínicos necesarios para resolver los problemas clínicos habituales ${ }^{14-19}$. Todo ello se adqui ere fundamentalmente a lo largo de los cuatro últimos cursos, pero culmina en el último año de la licenciatura en el que algunas asignaturas sólo tienen créditos prácticos. La lista de habilidades clínicas también será completada con el nivel de competencia requerido para un postgraduado ${ }^{17}$. El segundo paso del proceso consiste en definir los métodos docentes y de evaluación más i dóneos. Los resultados de esta autoevaluación ser án útil es en esteproceso in icial, pero nunca pueden sustituir a los obtenidos por un método objetivo y fiable de evaluación de habi lidades ${ }^{21-27}$.

El $83 \%$ de nuestros alumnos piensan que son capaces de realizar correctamente una anamnesis. Pensamos que este porcentaje es insuficiente en un grupo de postgraduados porque supone que un $17 \%$ de ellos no se encuentra capacitado para historiar pacientes. Los resultados de encuestas realizadas en otras facultades indican que el $97.5 \%$ de los alumnos contestan positivamente a esa cuestión ${ }^{13}$. Los porcentajes de adquisición de otras habilidades son todavía menores. Las del sistema respiratorio (52\%), sistema cardiovascular (46\%) y sistema cutáneo (41\%), que resultaron las mejor puntuadas, se adquirieron en un nivel menor al referido en la Universidad de Barcel ona $(80.6 \%, 72.1 \%$ y $87 \%$ respectivamente). El resto de habilidades obtuvieron puntuaciones inferiores al 30\%. Los resultados referidos a las habilidades psiquiátricas (8\%), también observados por otros autores ${ }^{13}$, pueden poner de manifiesto la dificultad de enseñarlas.

Estos resultados obligan a una reflexión en dos direcciones. La primera es si las habilidades definidas en esta encuesta deben ser exigibles a un médico general o deben ser reservadas a niveles de mayor competencia profesional relacionados con la formacoón especializada MIR. La segunda reflexión se refiere al método docente empleado en la enseñanza práctica. EI incremento de los oréditos prácticos ${ }^{28-30}$ debe acompañarse de un cambio de actitud global. EI nuevo concierto entre la UCO y las Instituciones Sanitarias será una excelente herramienta de mejora $^{31}$ aprovechando de forma integral hospitalización, consultas externas, urgencias y centros de salud ${ }^{32-38}$. En el futuro deberían reducirse los grupos de prácti cas integrándolos en la activi dad diaria de los servi cios asistenciales. Estas prácticas deberían completarse con la actividad en un laboratorio de habilidades $^{39-44}$. Todo $\mathrm{e}$ profesorado (titular y asociado) debe asumir la dedicación al alumno que exigen unas prácticas de calidad ${ }^{45,46}$. $L$ a dedicación de los catedráticos y profesores titulares a la gestión dínica debería ser compensada con profesores asociados. Posiblemente la competencia clínico-asistencial de los profesores vinculados debería ser más valorada para acceder a las pl azas docentes.

Finalmenteel alumno debe asumir la importancia que tiene la adquisición de habilidades dínicas para convertirse en un médico competente ${ }^{47}$. Entre las razones que explican su fal ta de interés están la incorrecta evaluación de los créditos prácticos y el examen MIR, que no las evalúa. Si sehici era, la evaluacoón debería tener una repercusión en la nota final de cada asignatura, proporcionada a sus créditos prácti cos. Los alumnos no se esforzarán en unas prácticas delas que son insuficientemente evaluados.

\section{BIBLIOGRAFÍA}

1. Leach DC. Competence is a habit. J AMA 2002; 287:243-4.

2. Dacre J E, Fox RA. How should we be teaching our undergraduates?. Ann Rheum Dis 2000;59:662-7

3. Barón M. La formación médica en Canadá como modelo de intervención profesional. Educación Médica 2001;4:52-65.

4. Epstein RM, Hundert EM. Defining and assessing professional competence. J AMA 2002;287:226-35

5. Campbell EJ. On education and training. Lancet 2000; 356:1116

6. Campbell J K, J ohnson C. Trend spotting: fashions in medical education. BMJ 1999;318:1272-5

7. Gjerde CL, Albanese M, Howard N. A faculty development program in basic teaching skills. Acad Med 1999;74:610-1

8. Spencer J A, J ordan RK. Learner centred approaches in medical education. BMJ 1999;318:1280-3.

9. Fowell SL, Bligh J G. Recent developments in assessing medical students. Postgrad Med J 1998;74:18-24

10. Margolis MJ, De Champlain AF, Klass DJ. Setting examination-level standars for a performance based assessment of physicians' clinical skills. Acad Med. 1998; 73 (suppl): S114S116.

11. Martínez Carretero J M, Blay Pueyo C. Evaluando la compe tencia de los profesionales sanitarios: dudas, fundamentos y experiencias. Educación Médica 1998; 1:17-23.

12. Simel DL, Rennie D. The clinical examination. An agenda to make it more rational. J AMA. 1997;277:572-4.

13. Palés J, Vallés A, Cardellach F, et al. Habilidades y procedimientos clínicos básicos a adquirir por los estudiantes de la Facultad de Medicina de la Universidad de Barcelona. Educación Médica 2001;4:72-81. 
14. ACGME Outcome Project (2000). Accreditation Council for Graduate Medical Education Web site. Disponible en: http://www.acgme.org

15. American Board of Internal Medicine. Internal Medicine blueprint. Disponible en http://www.abim.org.

16. The Scottish Dean's medical Curriculum Group. Learning outcomes for the Medical Undergraduate in Scotland: A foundation for competent and reflective practitioners. Disponible en: http://biology.st-and.acuk/scottishdoctor

17. Metz JCM, Stœlinga GBA, Pes EH, van den Brand BWM. Blueprint 1994: training of doctors in the netherlands. Objetives of undergraduate medical education. Universitair Publikatiebureau, University of Nij megen, The Netherlands 1994.

18. Goroll AH Morrison G, Bass EB, et al. Reforming the Core Clerkship in Internal Medicine: The SGIM/CDIM project. Ann Intern med 2001;134:30-7.

19. Barritt AS 3rd. Reforming the core clerkship in internal medicine. Ann Intern Med. 2001;135:635.

20. Blanchard K, Carlos J P, Randolph A. El empowerment requiere más de un minuto. Ediciones Deusto. Bilbao, 1996.

21. van der Vleuten $C$. Validity of final examinations in undergraduate medical training. BMJ . 2000; 321: 1217-9.

22. Wass V, J ones $R$, van der Vleuten $C$. Standardized or real patients to test clinical competence? The long case revisited. Med Educ. 2001; 35: 321-5.

23. McManus IC, Richards P, Winder BC, Sproston KA. Clinical experience, perfomance in final examinations, and learning style in medical students: prospective study. BMJ 1998; 316:345-50.

24. J olly B, Murray E. Clinical experience and performance in final examinations. Valid methods are needed to assess students. BMJ 1998;316:1984-5.

25. Kassebaum DG, Eaglen RH. Shortcomings in the evaluation of students' dinical skills and behaviors in medical school. Acad Med. 1999; 74: 842-9.

26. Battistone MJ , Pendleton B, Milne C, Battistone ML, Sande MA, Hemmer PA, Shomaker TS. G lobal descri ptive eval uations are more responsive than global numeric ratings in detecting students' progress during the inpatient portion of an internal medi ane derkship. Acad M ed. 2001;76(10 Suppl):S105-7.

27. Ramsey PG, Wenrich MD, Carline J D, Inui TS, Larson EB, LoGerfo J P. Use of peer ratings to evaluate physician performance. J AMA. 1993;269:1655-60.

28. Resolución 28 de Noviembre de 1995, de la Universidad de Córdoba, por la que se ordena la publicación del plan de estudios del título de Licenciado en Medicina, a impartir en la Facultad de Medicina de esta universidad. BOE no 30 de 3 de Febrero de 1996.

29. Resolución de 23 de Mayo de 1996, de la Universidad de Córdoba, por la que se corrigen errores en la de 28 de
Noviembre de 1995, por la que se publicaba el plan de estudios del título de Licenciado en Medicina. BOE no 143 de 13 de J unio de 1996.

30. Resolución de 19 de Julio de 2000 , de la Universidad de Córdoba, por la que se modifica el plan de estudios de Licenciado en Medicina.

31. Resolución de 31 de Julio de 2000, de la Universidad de Córdoba, por la que se da publicidad al Concierto entre la J unta de Andalucía y la Universidad de Córdoba para la utilización de las Instituciones Sanitarias en Ia Investigación y la Docencia. BOJ A 102 de 5 de Septiembre del 2000.

32. Anderson RC, Fagan MJ , Sebastian J. Teaching students the art and science of physical diagnosis. Am J Med 2001; 110:419-23.

33. Devitt N. The rational dinical examination. JAMA. 1992; 268:2164.

34. F ields SA, U satine R, Steiner $E$. Teaching medical students in the ambulatory setting: strategies for success. J AMA 2000 May 10;283:2362-4.

35. Kernan WN, Lee MY, Stone SL, Freudigman KA, O'Connor PG. Effective teaching for preceptors of ambulatory care: a survey of medical students. Am J Med 2000;108:499-502.

36. Lamb MG. The rational dinical examination. J AMA. 1992;268:2165.

37. Reznek M, Harter P, Krummel T. Virtual reality and simulation: training the future emergency physician. Acad Emerg Med 2002;9:78-87.

38. Schatz IJ . Training more generalists. Ann Intern Med. 1994; 120:92.

39. Pololi LH. Standardised patients: as we evaluate, so shall we reap. Lancet 1995;345:966-8.

40. Bardes CL. Evaluating the curriculum with standardized patients. Acad Med 1998;73:626-7.

41. Blay Pueyo C. Evaluación clínica objetiva y estructurada: ECOE. Educación Médica 1998; 1:13-6.

42. Colliver J A, Swartz MH. Assessing clinical performance with standardized patients. J AMA. 1997; 278: 790-1.

43. Gomez J M, Prieto L, Pujol R, et al. Clinical skills assessment with standardized patients. Med Educ. 1997; 31: 94-8.

44. Kaufman DM, Mann KV, Muijtjens AM, van der Vleuten C. A comparison of standard-setting procedures for an OSCE in undergraduate medi cal educati on. Acad M ed. 2000; 75: 267-71.

45. Asch E, Saltzberg D, Kaiser S. Reinforcement of self-directed learning and development of proffesional attitudes through peer- and self-assessment. Acad Med. 1998;73:575.

46. Phillips LS, Branch WT, Cook CB, Doyle J P, EI-Kebbi IM, Gallina DL, Miller CD, Ziemer DC, Barnes CS. Clinical inertia. Ann Intern Med 2001;135:825-34.

47. Leung WC. Teaching styles need to be reviewed to help students with inappropiate learning styles. BMJ 1998;316:1984. 